\title{
Estudio bibliométrico de la producción científica sobre TIC en Perú (2010-2017)
}

\section{Bibliometric Study of Scientific Production on ICT in Perú (2010-2017)}

\author{
Nicanor Segismundo Asmat Vega \\ Universidad Nacional Federico Villarreal, Lima, Perú \\ ORCID: https://orcid.org/0000-0003-0087-6515
}

César Andrés Borja Villanueva*

Universidad Privada Juan Pablo II, Lima, Perú

ORCID: https://orcid.org/0000-0002-4274-4978

Luis Alexis Bernuy Torres

Universidad Privada Juan Pablo II, Lima, Perú

ORCID: https://orcid.org/0000-0001-9011-7699

Danny Alonso Lizarzaburu Aguinaga

Universidad Cesar Vallejo, Lima, Perú

ORCID: https://orcid.org/0000-0002-1384-4603

John Morillo Flores

Universidad Continental, Lima, Perú

ORCID: https://orcid.org/0000-0002-2136-4458

Recibido 12-08-18 Revisado 30-09-18 Aprobado 17-12-18 En línea 19-12-18

*Correspondencia

Email: abv1979@gmail.com
Citar como:
Asmat, N., Borja, C., Bernuy, L., Lizarzaburu, D., \& Morillo, J. (2019). Estudio bibliométrico de la producción científica sobre TIC en Perú (2010-2017). Propósitos y Representaciones, $\quad 7(2), \quad$ 196-209. doi: http://dx.doi.org/10.20511/pyr2019.v7n2.269 


\section{Resumen}

La presente investigación tiene como objetivo evaluar la producción científica peruana en las Tecnologías de Información y Comunicación (TIC). Es un estudio descriptivo transversal mediante el análisis bibliométrico de los artículos publicados en revistas indizadas en SciELO Perú entre el año 2010 y 2017. Se analizaron autoría, tipo de artículo, ejes temáticos, y productividad institucional. Se encontró que entre los años 2010 y 2017, se ha publicado 19 artículos sobre TIC, de los cuales el 26.4\% son publicaciones en la Revista Peruana de Medicina Experimental y Salud Publica, y el 30.8\% de los autores indican tener como afiliación principal a la Universidad Nacional Mayor de San Marcos. El principal eje temático en el conjunto de publicaciones fue la enseñanza virtual (26\%). Podemos concluir que el desarrollo tecnológico viene contribuyendo con la publicación científica del país; sin embargo, es necesario seguir fortaleciendo estos estudios en demás bases de datos.

Palabras clave: Producción científica; TIC; SciELO Perú.

\section{Summary}

The objective of this research is to evaluate Peruvian scientific production in Information and Communication Technologies (ICTs). It is a cross-sectional descriptive study through the bibliometric analysis of articles published in journals indexed in SciELO Peru between 2010 and 2017. Authorship, type of article, main themes, and institutional productivity were analyzed. It was found that between 2010 and 2017, 19 articles on ICT were published, 26.4\% of them are publications in the Peruvian Journal of Experimental Medicine and Public Health, and 30.8\% of authors indicate that their main affiliation is with the National University of San Marcos. The main theme in the set of publications was virtual teaching (26\%). We can conclude that technological development is contributing to the scientific publication of the country; however, it is necessary to continue strengthening these studies in other databases.

Keywords: Scientific Production; ICT; SciELO Peru.

\section{Introducción}

La Tecnología de Información y Comunicación (TIC) y su implicancia en la investigación ha pasado por diversas etapas, en las que se han producido cambios tanto en los problemas de investigación planteados como en la metodología utilizada. El aumento de la producción de trabajos (en forma de libros colectivos, de artículos en revistas, de documentos electrónicos en la Red, de comunicaciones a Congresos, de informes de investigación, etc.) es un hecho palpable (Vidal, 2006; Morales Trujillo \& Raso, 2015).

En el Perú el proceso de integración de las nuevas TIC al sistema educativo se genera a través del Proyecto Huascarán y actualmente a través de la Dirección de Tecnologías Educativas, quien define tres capacidades que debe implementarse en el contexto educativo 1) Información.La adquisición de información, donde los estudiantes investigan más y mejor con las TIC; 2) Comunicación.- El trabajo en equipo con las TIC, donde los estudiantes consolidan el trabajo cooperativo y eficiente en cada una de las áreas curriculares y 3) Producción.- El desarrollo de estrategias de aprendizaje a través de la producción de materiales educativos con TIC (Larrauri, 2009; Rivoir, 2016). 
En el mundo, a partir de la evolución y auge tecnológico en las ciencias de la información, se vienen dando grandes cambios en la sociedad actual. En el contexto de la cienciométria, los estudios de visibilidad se realizan con frecuencia con el objetivo de determinar la presencia de la producción científica de un país en una o varias bases de datos internacionales de gran prestigio y utilidad reconocida, en una o varias disciplinas (Cañedo et al., 2014) constituyéndose en el núcleo fundamental de la evaluación en la actividad investigadora (Giménez-Toledo, 2015). Esto ha llevado a que las universidades se preocupen en observar el incremento en sus publicaciones en los últimos años (Gonzales-Argote, García-Rivero y Dorta-Contreras, 2016).

Las revistas científicas sin duda son los principales canales de comunicación y difusión de los resultados de investigación y de institucionalización social de la ciencia en la mayoría de los campos del conocimiento. La necesidad de comunicación entre los investigadores es una de las razones que mantiene a estos medios en constante evolución, siendo los artículos científicos la principal moneda de cambio ya que esta refleja el capital simbólico detentado por los investigadores (Kreimer, 1998).

El objetivo de la presente investigación fue describir el desarrollo de la producción científica sobre TIC, realizadas en Perú, lo que ayudará a conocer las fortalezas, capacidades y limitaciones de esta variable de investigación en nuestro medio.

\section{Método}

Se realizó un estudio bibliométrico que consistió en la búsqueda, extracción y análisis de publicaciones científicas enmarcados en las Teconologías de Información, que contaran con al menos un autor con filiación a una institución peruana o que hubiera involucrado población peruana, durante el periodo 2010-2017. La búsqueda fue realizada en la base de datos SciELO PERÚ. Se realizó la búsqueda en el título, resumen y palabras clave empleando términos de búsqueda, añadiendo un filtro para el periodo 2010 al 2017. Se recuperó 19 publicaciones de revistas indizadas en SciELO Perú.

\section{Resultados}

Con respecto al número de publicaciones por año, se observa una equidad de contribuciones, manteniéndose un promedio de 1 a 2 artículos por año, y se evidencia, además, un aumento en las publicaciones en el año 2015 ( $n=9$ ), ver figura 1.

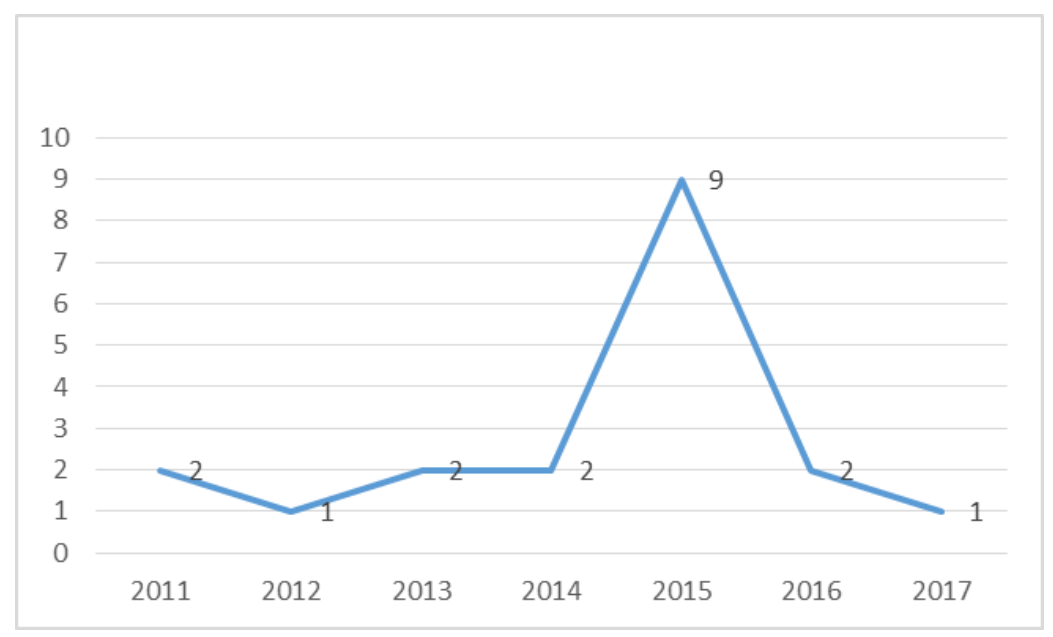


Figura 1. Estimación de las publicaciones por año.

Los artículos analizados fueron publicados en 10 revistas. La Tabla 1 muestra las revistas donde se han publicado los artículos con referencia a las TIC, el $26.3 \%$ de las publicaciones, se encuentran en la Revista Peruana de Medicina Experimental y Salud Publica, seguido de la Revista Médica Herediana y de la Revista de Psicología (PUCP) (15.8\%), ver tabla 1.

\section{Tabla 1.}

Revistas donde se publican los artículos científicos de TIC.

\begin{tabular}{lccc}
\hline \multicolumn{1}{c}{ Revistas } & $\mathrm{N}$ & $\%$ & $\mathrm{H}$ Index \\
& & & SJR \\
\hline Revista Peruana de Medicina Experimental y Salud Publica & 5 & 26.3 & 14 \\
Revista Médica Herediana & 3 & 15.8 & $\mathrm{ND}$ \\
Revista de Psicología (PUCP) & 3 & 15.8 & 1 \\
Anales de la Facultad de Medicina & 2 & 10.5 & $\mathrm{ND}$ \\
Revista de Gastroenterología del Perú & 1 & 5.3 & 14 \\
Acta Médica Peruana & 1 & 5.3 & $\mathrm{ND}$ \\
Comuni @ cción & 1 & 5.3 & $\mathrm{ND}$ \\
Horizonte Médico & 1 & 5.3 & $\mathrm{ND}$ \\
Revista Peruana de Ginecología y Obstetricia & 1 & 5.3 & $\mathrm{ND}$ \\
Journal of Economics, Finance and Administrative Science & 1 & 5.3 & 6 \\
& & & \\
\hline
\end{tabular}

En 19 artículos se pudo identificar a los autores con afiliación a instituciones nacionales, destacando a la Universidad Nacional Mayor de San Marcos (30.8\%) seguido de la Universidad Peruana Cayetano Heredia (17.9\%), La Tabla 2 muestra a los autores con afiliación a instituciones nacionales con publicaciones sobre TIC. 


\section{Tabla 2.}

Instituciones nacionales que han publicado artículos sobre TIC.

\begin{tabular}{lll}
\hline \multicolumn{1}{c}{ Instituciones } & $\mathrm{N}$ & $\%$ \\
\hline Universidad Nacional Mayor de San Marcos & 12 & 30.8 \\
Universidad Peruana Cayetano Heredia & 7 & 17.9 \\
Pontificia Universidad Católica del Perú & 4 & 10.3 \\
Sociedad Peruana de Obstetricia y Ginecología & 2 & 5.1 \\
Universidad Nacional José Faustino Sánchez Carrión & 2 & 5.1 \\
Instituto Nacional de Enfermedades Neoplásicas (INEN) & 1 & 2.6 \\
Seguro Social de Salud, EsSalud & 1 & 2.6 \\
Universidad Nacional del Altiplano & 1 & 2.6 \\
Hospital Nacional Cayetano Heredia & 1 & 2.6 \\
$\begin{array}{l}\text { Consejo Nacional de Ciencia, Tecnología e Innovación Tecnológica } \\
\text { (CONCYTEC) }\end{array}$ & 1 & 2.6 \\
Sociedad Científica de Estudiantes de Medicina Cayetano Heredia & 1 & 2.6 \\
Universidad de Ciencias y Humanidades & 1 & 2.6 \\
Hospital III de Emergencia Grau & 1 & 2.6 \\
Oficina de las Naciones Unidas de Servicios para Proyectos (UNOPS). & 1 & 2.6 \\
Health Care Consulting S.A.C. & 1 & 2.6 \\
Universidad ESAN & 1 & 2.6 \\
Universidad de San Martín de Porres & 1 & \\
\hline & & 1 \\
\hline
\end{tabular}

El $26 \%$ de artículos correspondió a variables relacionadas a la "enseñanza virtual” (26\%), seguido de educación médica y salud pública con un $21 \%$ respectivamente. Ver figura 2 :

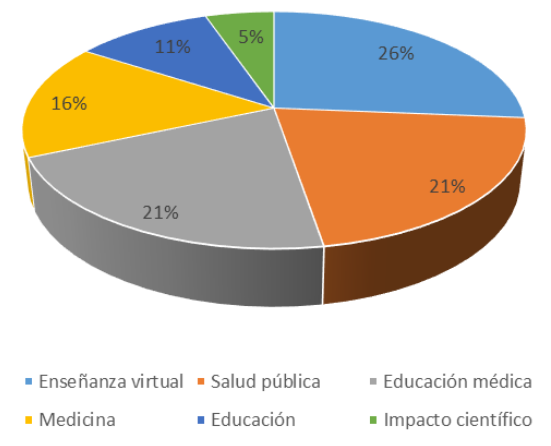

Figura 2. Temáticas de mayor incidencia en publicación de TICs. 


\section{Discusión}

La evaluación del impacto de las TIC es una de las cuestiones fundamentales a abordar en las siguientes décadas (Gargallo et al., 2004). Es necesario fortalecer las investigaciones en contextualizadas en los diferentes campos de estudio (Linares-Pons, Verdecia-Martínez, \& Álvarez-Sánchez, 2014).

Este artículo es un análisis descriptivo acerca de las publicaciones sobre TIC realizadas en el Perú entre los años 2010 - 2017. Se ha observado un incremento sostenido en la producción literaria y es En el año 2015 donde se ve un marcado incremento en la producción científica sobre TIC. Sin embargo, en producción total, el número de publicaciones sobre TIC en el Perú es mucho menor a la descrita en España (Buceta, Martínez \& Páramo, 2016; Aznar, Trujillo \& Romero, 2018), Colombia (Muñoz, Bolívar \& Hernández, 2017).

Como potenciales limitaciones del estudio están relacionadas a las bases de datos utilizadas, existiendo otras investigaciones que han sido publicadas en otras revistas internacionales indizadas otras bases. Sin embargo, consideramos la importancia de poder revisar publicaciones nacionales en revistas nacionales, lo que evidenciaría la participación de investigadores de nuestro medio en dar a conocer los resultados de sus investigaciones en el medio local.

A pesar de las limitaciones (cantidad de autores y coautores; si eran estudiantes de pregrado, posgrado o profesionales, factor de impacto de las publicaciones) se considera que los hallazgos obtenidos aportan a un mejor conocimiento sobre la producción científica en las ciencias del deporte.

A pesar de que la producción científica sobre TIC se ha incrementado en los últimos años, el volumen de producción en el Perú es bajo en comparación con países desarrollados y otros en vías de desarrollo (Mominó, \& Sigalés, 2017). La investigación que se hace es básicamente abarca el campo de la educación, a través de la enseñanza-aprendizaje virtual. La participación de las Instituciones nacionales es un referente, porque nos muestra las diferencias existentes ente universidades, hospitales, consultoras que vienen implementado investigación en TIC, durante los últimos 10 años. Y se recomienda que se realicen estudios bibliométricos en otros continentes y que consideren intervalos de años mayor o igual al presente estudio, como también analizar las aplicaciones disponibles a través de las TIC (Buitrago, García, \& García, 2016).

\section{Referencias}

Aznar Díaz, I., Trujillo Torres, J. M., \& Romero Rodríguez, J. M. (2018). Estudio bibliométrico sobre la realidad virtual aplicada a la neurorrehabilitación y su influencia en la literatura científica. Revista Cubana de Información en Ciencias de la Salud, 29(2). Recuperado de: http://acimed.sld.cu/index.php/acimed/article/view/1209

Buceta Vicente, L., Martínez Figueira, M. E., \& Páramo Iglesias, M. B. (2016). Iniciando un estudio bibliométrico sobre las potencialidades de las TIC en el aprender a aprender en Educación Infantil. Recuperado de: https://idus.us.es/xmlui/handle/11441/43657

Buitrago, L. D. F., García, C. R., \& García, S. R. (2016). Las Tic como herramientas de inclusión social. 3C TIC, 5(1), 54-67. Doi: https://doi.org/10.17993/3ctic.2016.51.54-67 
Cañedo Andalia, R., Nodarse Rodríguez, M., Pupo, J. C. G., Muñoz, I. A., Chapman, M. C. S., \& Ramírez, L. J. M. (2014). Producción científica en salud de Cuba en bases de datos internacionales. Revista Cubana de Información en Ciencias de la Salud (ACIMED), 25(4), 442-451. Recuperado de: http://acimed.sld.cu/index.php/acimed/article/view/584

Gargallo, B., Suárez, J., Morant, F., Marín, J. M., Martínez, M., \& Díaz, I. (2004). Un primer diagnóstico del uso de internet en los centros escolares de la Comunidad Valenciana. Procesos de formación y efectos sobre la calidad de la educación. Valencia: IVECE (Instituto Valenciano de Evaluación y Calidad Educativa).

Giménez-Toledo, E. (2015). La evaluación de la producción científica: breve análisis crítico. Relieve. Revista electrónica de investigación y evaluación educativa, 21(1), 1- 9. Doi: https://doi.org/10.7203/relieve.21.1.5160

Gonzalez-Argote, J., Garcia-Rivero, A. A., \& Dorta-Contreras, A. J. (2016). Producción científica estudiantil en revistas médicas cubanas 1995-2014. Primera etapa. Investigación en educación médica, 5(19), 155-163. Doi: http://dx.doi.org/10.1016/j.riem.2016.01.023

Kreimer, P. (1998). Publicar y castigar. El paper como problema y la dinámica de los campos científicos. Redes, (12), 253.-258. Recuperado de: https://www.redalyc.org/html/907/90711317002/

Larrauri, R. C. (2009). Eficacia en el desarrollo de capacidades TIC en estudiantes de educación secundaria de Lima, Perú. Pixel-Bit. Revista de Medios y Educación, (35), 5-20. Recuperado de: https://recyt.fecyt.es/index.php/pixel/article/view/61348

Linares-Pons, N., Verdecia-Martínez, E. Y., \& Álvarez-Sánchez, E. A. (2014). Tendencias en el desarrollo de las TIC y su impacto en el campo de la enseñanza. Revista Cubana de Ciencias Informáticas, $8(1), \quad 71-78 . \quad$ Recuperado de: http://rcci.uci.cu/?journal=rcci\&page=article\&op=view\&path\%5B $\% 5 \mathrm{D}=517$

Mominó, J. M., \& Sigalés, C. (2017). El impacto de las TIC en la educación. Más allá de las promesas. Editorial UOC.

Morales Capilla, M., Trujillo Torres, J. M., \& Raso Sánchez, F. (2015). Percepciones acerca de la integración de las TIC en el proceso de enseñanza-aprendizaje de la universidad. PixelBit. Revista de Medios y Educación, 46, 103-117. Recuperado de: https://idus.us.es/xmlui/handle/11441/45403

Muñoz, L. A., Bolívar, M. P. R., \& Hernández, A. M. L. (2017). Análisis Bibliométrico sobre la Implementación de las TICs en las Administraciones Públicas: Aportaciones y Oportunidades de Investigación. Innovar, 27(63), 141-160. Doi: https://doi.org/10.15446/innovar.v26n63.60674

Rivoir, A. (2016). Revisión comparativa de iniciativas nacionales de aprendizaje móvil en América Latina: el caso de la política TIC en el Perú.

Vidal, Ma.P. (2006). Investigación de las TIC en la educación, Revista Latinoamericana de Tecnología Educativa, 5(2), 539-552. Recuperado de: http://dehesa.unex.es/handle/10662/1436 